\title{
Recurrent Unilateral Nasal Obstruction: A Cause Lying Deep Inside
}

\section{Soumyadeep Biswas ${ }^{1}$, Shatanik Sarkar ${ }^{2}$, Debasish Bandyopadhyay ${ }^{1}$ and Chaitali Patra $^{3}$}

${ }^{1}$ Department of Paediatrics, B. R. Singh Hospital, India

${ }^{2}$ Department of Paediatrics, R. G. Kar Medical College, India

${ }^{3}$ Department of Paediatrics, Tapan Sinha Memorial Hospital, India

\author{
Correspondence: \\ Shatanik Sarkar, \\ Department of Paediatrics, \\ R. G. Kar Medical College, India \\ Deshbandhunagar, Baguiati, Kolkata \\ Mobile No. +919433543732 \\ Email Id - baselinegame@gmail.com
}

DOI: $10.3126 /$ jnps.v39i2.26911

Submitted on: $2019-12-27$

Accepted on: 2020-03-14

Acknowledgements: None

Funding: Nil

Conflict of Interest: None declared

Permission form IRB: Yes

To cite this article: Biswas $\mathrm{S}$, Sarkar S, Bandyopadhyay D, Patra C. Recurrent Unilateral Nasal Obstruction: A cause lying deep inside. J Nepal Paediatr Soc. 2019;39(2):123-6.

\begin{abstract}
Recurrent nasal obstruction, a common symptom in pediatric practice, can lead to some unusual diagnosis rarely. Nonresponse to standard treatment warrants further delineation of local anatomy, particularly if symptoms occur unilaterally. Here, we report a case of recurrent unilateral nasal obstruction in a 16 years old girl not amenable to conventional treatment with medicines, which on subsequent investigations revealed an impacted intranasal tooth.
\end{abstract}

Key words: Foreign Body; Intranasal tooth; Nasal obstruction; Rhinoscopy 


\section{INTRODUCTION}

Recurrent nasal obstruction is a reasonably common entity in paediatric population. Common causes of recurrent nasal obstruction are allergic or non-allergic rhinitis, sinusitis, deviated nasal septum, nasal polyp etc. When nasal obstruction is unilateral and not responding to usual treatment, a local cause has to be searched for with examination and investigations to delineate the deeper anatomy of the nasal cavity. Here we present a case with recurrent unilateral nasal obstruction, ultimately diagnosed as a case of impacted intranasal teeth.

Ectopic intranasal teeth are rare and can occur in different locations. Ectopic teeth are commonly seen in palate, maxillary sinus and the floor of the nasal cavity. In children, intranasal ectopic teeth occur more frequently in patients with cleft lip and alveolus. ${ }^{1}$ In this article, we report an unusual and rare case of an intranasal ectopic tooth in a child without any obvious etiology and unusual presentation.

\section{CASE REPORT}

A 16 years old adolescent female presented at the outpatient department of our hospital with history of recurrent left sided nasal obstruction interspersed with occasional symptoms of nasal discharge and heaviness over the left side of face for last three months. During the initial visits she was prescribed with anti-histaminics which provided some amount of symptomatic relief to the patient, but the symptoms recurred within a few days.

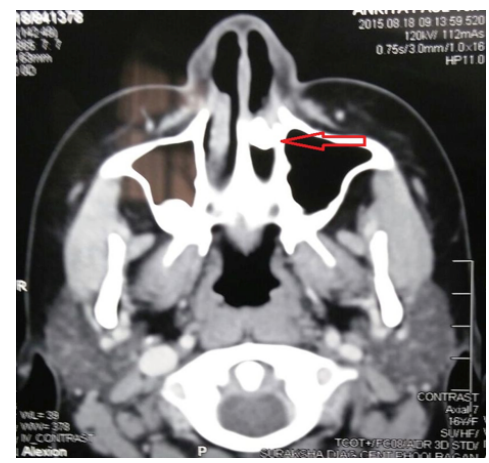

Figure1. CECT Scan showing presence of impacted foreign body in the left nasal cavity (arrowhead)
Thorough general and systemic examination didn't reveal any significant abnormality. There was no local swelling or tenderness. On anterior rhinoscopy, a creamy white mass with surrounding granulation tissues was noted deep into the left nasal cavity along the floor. No abnormality was detected in posterior rhinoscopy. Her intraoral dentition was normal with no cleft palate or other congenital anomalies involving the perioral structures. No previous history of maxillofacial trauma, surgery or nasal foreign body was elicited. Contrast Enhanced Computed Tomography (CECT) Scan done in our hospital showed a radiopaque mass located in the left nasal cavity between the inferior turbinate and the nasal septum.

Trial of removal of the foreign body by endoscopic techniques not only resulted in failure owing to its location deep into the nasal cavity, but also resulted in heavy epistaxis. The patient was subsequently planned for open surgical intervention after epistaxis was controlled conservatively. Exploraion under general anaesthesia was undertaken at a later date and the foreign body was removed from the left nasal cavity. On removal, the foreign body could be identified as an intranasal tooth (Figure 2). The patient was discharged after her hospital stay and regularly followed up at the out-patient department, but her previous symptoms were not a cause of concern any further.

\section{DISCUSSION}

The presence of an ectopic tooth within the nasal cavity is a very rare phenomenon with a male predominance and around half of all patients are

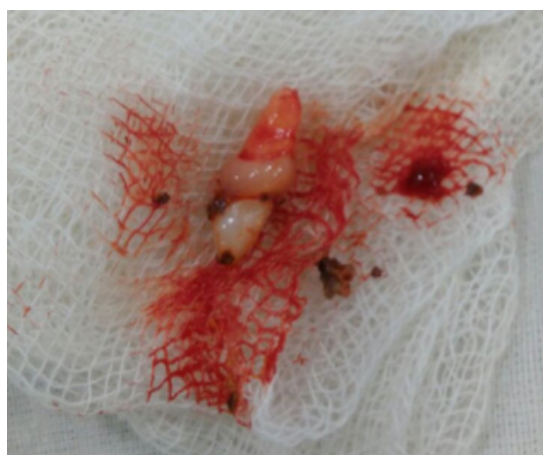

Figure 2. The tooth after extraction 
diagnosed before adulthood. It occurs only in 0.1 to $1 \%$ of normal population. ${ }^{2}$ The most common location of an ectopic tooth is the upper incisor area, known as mesiodens. ${ }^{3}$ But, it may be present in many regions of the maxillofacial skeleton, including palate, nose, orbit, maxillary sinus, and also as teratomas in ovary, testes, anterior mediastinum etc. Ectopic intranasal tooth arising from inferior turbinate is very rare. From 1966 to 2014 , ectopic tooth arising from inferior turbinate in two adult female skulls has been reported by Ray et al. ${ }^{4}$ and in a paediatric patient by Yu et al. ${ }^{5}$

Intranasal tooth is a rare event that can occur due to some trauma, anatomical malformation or genetic factors. It has been proposed that supernumerary teeth develop either from a third tooth bed that arises from the dental lamina near the permanent tooth bud or, possibly, from splitting of the permanent bud itself. ${ }^{6}$ Another theory is that there is a defect in the migration of neural crest derivatives destined to reach the jawbones. ${ }^{7}$ They are generally associated with other conditions like cleft palate, maxillofacial trauma, Gardner's syndrome and cleidocranial dysostosis. ${ }^{5}$ But, no such anomaly was noted in our case.

The extra tooth usually has an atypical crown and may be aligned in a vertical, horizontal or inverted position ${ }^{9}$. Such an extra tooth, when present intranasally, can be completely asymptomatic or can present with nasal obstruction, epistaxis, headache, foul smelling nasal discharge, external nasal deviation, nasolacrimal duct obstruction or oro-nasal fistula formation. ${ }^{8}$

The diagnosis of nasal teeth is mainly based on clinical and radiographic examination. Clinically, an intranasal tooth may be completely or incompletely embedded in the nasal mucosa, and when diagnosed, its early extraction is of paramount importance because of the potential morbidity associated with it.
Differential diagnosis of an ectopic nasal tooth includes foreign body, rhinoliths, granulomatous infections and tumours. ${ }^{3}$ The CT findings of toothequivalent attenuation and a centrally located cavity are highly discriminating features that help to confirm the diagnosis. ${ }^{3}$

The treatment of intranasal teeth is early surgical extraction to alleviate the symptoms and prevent the possible morbidities that includes rhinosinusitis, osteomyelitis, dacryocystitis, nasal septal abscess or perforation, oronasal or intraoral fistula, aspergillosis and nasal deformity. 3,8

Although endoscopic removal of intranasal tooth in some cases have shown encouraging results, but when a tooth has a bony socket in the floor of the nose, it may be extremely difficult to extract. ${ }^{9}$ In case of submucosal foreign bodies between the nasal mucosa and the nasal septal cartilage and those that have been present for some time and have become encrusted or impacted, open surgical techniques might be required ${ }^{10}$ as in our case.

\section{CONCLUSIONS}

Unilateral nasal obstruction recurring repeatedly without satisfactory relief after medications can have causes lying deep inside. Intranasal teeth are a rare form of ectopic teeth causing a variety of symptoms and complications. Early diagnosis and treatment are very important to avoid the dreaded complications associated with it. Furthermore, intranasal supernumerary tooth in a female adolescent without any obvious cause or associated anomalies is a rare entity.

\section{REFERENCES}

1. Yeung K.H., Lee K.H. Intranasal tooth in a patient with a cleft lip and alveolus. Cleft Palate Craniofac J. 1996;33:157-9. DOI: https://doi.org/10.1597/1545-1569_1996_033_0157_itiapw_2.3.co_2

2. Pracy JP, Williams HO, Montgomery PQ. Nasal teeth. J Laryngol Otol. 1992;106:366-7. DOI: https://doi.org/ $10.1017 / \mathrm{S} 0022215100119516$ 
3. Chen A, Huang JK, Cheng SJ, Sheu CY. Nasal Teeth: Report of Three Cases. Am J Neuroradiol. 2002;23:671-3. PMID:11950665

4. Ray B, Singh LK, Das CJ. Ectopic supernumerary tooth on the inferior nasal concha. Clin Anat. 2006;19:68-74. DOI: https://doi.org/10.1002/ca.20161

5. Chao Y. Case presentation of an intranasal ectopic tooth in a pediatric patient. Am J Otolaryngol. 2015;36:472-4. DOI: https://doi.org/10.1016/j.amjoto.2015.01.020

6. Thawley SE, Ferriere KA. Supernumerary nasal tooth. Laryngoscope. 1977;87:1770-3. DOI: https://doi.org/ $10.1288 / 00005537-197710000-00025$

7. AlDhafeeri HO, Kavarodi A, Shaikh KA, Bukhari A, Hussain OA, Baramawy AE. Recurrent epistaxis caused by an intranasal supernumerary tooth in a young adult. Am J Case Rep. 2014;15:291-3. DOI: https://doi.org/10.12659/ AJCR.890710

8. Alexandrakis G., Hubbell R.N., Aitken P.A. Nasolacrimal duct obstruction secondary to ectopic teeth. Ophthalmol. 2000;107:189-92. DOI: https://doi.org/10.1016/S0161-6420(99)00023-8

9. Wurtele P, Dufour G. Radiology case of the month: a tooth in the nose. J Otolaryngol. 1994;23:67-8. PMID: 8170025

10. Patil PM, Anand R. Nasal foreign bodies: A review of management strategies and a clinical scenario presentation. Craniomaxillofac Trauma Reconstr. 2011;4:53-8. DOI: https://doi.org/10.1055/s-0031-1272902 